\title{
Spinach Tissue Culture Improved with Coconut Water
}

\author{
Jameel M. Al-Khayri, Feng H. Huang, Teddy E. Morelock, and \\ Tahani A. Busharar \\ Department of Horticulture and Forestry, University of Arkansas, \\ Fayetteville, AR 72701
}

Additional index words. callus, leaf explant, micropropagation, regeneration, Spinacia oleracea

\begin{abstract}
A preliminary study has shown that the addition of $15 \%$ (v/v) coconut water (CW) to the culture medium significantly improved callus growth, shoot-regenerative capacity, and shoot growth in leaf disk cultures of spinach (Spinacia oleracea L.). Subsequently, the influence of a range of $\mathrm{CW}$ concentrations, $0 \%, 5 \%, 10 \%, 15 \%$, or $20 \%(v / v)$, was examined. Callus weight obtained after 5 weeks showed direct relationship to the concentration of CW. This stimulator action was observed in both cultivars tested in this study, 'High Pack' and 'Baker'. On CW-containing medium, shoot regeneration was expedited to 4 to 5 weeks compared with 8 to 12 weeks on a CW-free medium. Callus of 'Baker' induced on a CW-free medium exhibited a significant increase in shoot regeneration frequency when transferred to a regeneration medium enriched with $\mathrm{CW}$, suggesting that the addition of $\mathrm{CW}$ to the regeneration medium only is sufficient to achieve improved regeneration.
\end{abstract}

Recent studies established a tissue-culture system for the plant regeneration of spinach from leaf-derived callus (Al-Khayri et al., 1991a, 1991 b). Improvement in the percentage of spinach calli forming shoots had been desired because of the low regeneration frequency. Our objectives were to 1) examine the influence of various concentrations of coconut water $(\mathrm{CW})$ on callus induction and subsequent shoot regeneration, and 2) determine whether limiting the enrichment of $\mathrm{CW}$ only to the regeneration medium would improve shoot formation.

Plant material. Seeds of 'Baker' and 'High Pack' spinach were planted in potting soil (Fisons Sunshine mix no. 1, Vancouver, B. C.) and grown in a growth chamber set to provide $10-\mathrm{h}$ photoperiods (350 $\left.\mu \mathrm{mol} \cdot \mathrm{m}^{-2} \cdot \mathrm{s}^{-1}\right)$ and day/night cycles of $20 \mathrm{C} /$ 16C. Leaves 5 to $7 \mathrm{~cm}$ long were surface sterilized in $70 \%$ ethanol for 30 see, followed by immersion for $5 \mathrm{~min}$ in $1 \%$ sodium hypochlorite containing $0.01 \%(\mathrm{v} / \mathrm{v})$ Tween 20 , and rinsed three times in sterile distilled water. Leaf disk explants $5 \mathrm{~mm}$ in diameter were isolated with a cork borer.

Callus induction. Leaf disks were cultured on medium consisting of Murashige and Skoog (MS) basal salts (Murashige and Skoog, 1962) augmented with (in mg.liter ${ }^{-1}$ ) 100 myo-inositol, 0.4 thiamine-HC1, 0.5 nicotinic acid, 0.5 pyridoxine- $\mathrm{HC} 1,9.3 \mu \mathrm{M} \mathrm{N}-$ (2-furanylmethyl-1H-purine-6-amine) (kinetin), $2.3 \mu \mathrm{M}$ 2,4-dichlorophenoxyacetic acid $(2,4-\mathrm{D})$, and $30 \mathrm{~g}$ sucrose/liter. The medium

Received for publication 23 Sept. 1991. Accepted for publication 16 Dec. 1991. Published with approval of the Director of Arkansas Agricultural Experiment Station. The cost of publishing this paper was defrayed in part by the payment of page charges. Under postal regulations, this paper therefore must be hereby marked advertisement solely to indicate this fact. was adjusted to $\mathrm{pH} 5.8$ and solidified with 8 g tissue culture agar/liter (agar-agar/gum agar, Sigma, St. Louis). Filter-sterilized CW, extracted from mature nuts purchased at a local supermarket, was added at $0 \%, 5 \%, 10 \%, 15 \%$, or $20 \%(\mathrm{v} / \mathrm{v})$ to partially cooled autoclaved (121C, $15 \mathrm{~min})$ medium. Media were dispensed in $16 \times 100-\mathrm{mm}$ culture tubes $(5 \mathrm{ml} /$ tube). One leaf disk was used per tube, and the cultures were incubated at $20 \pm 5 \mathrm{C}$ in darkness. At the end of 4 weeks, callus weights were obtained.

Shoot regeneration. Four weeks after culture initiation, 18 calli per treatment were transferred onto regeneration medium. The regeneration medium consisted of the same components described for callus induction medium, including CW concentrations, but 2,4-D was reduced to $0.05 \mu \mathrm{M}$, and $2.9 \mu \mathrm{M}$ gibberellic acid $\left(\mathrm{GA}_{3}\right.$ was added. Media were dispensed in $25 \times 150-\mathrm{mm}$ tubes $(15 \mathrm{ml} /$ tube) and cultures maintained at $20 \pm 5 \mathrm{C}$ and exposed to a 10-h photoperiod (50 $\left.\mu \mathrm{mol} \cdot \mathrm{m}^{-2} \cdot \mathrm{s}^{-1}\right)$ supplied by cool-white fluorescent tubes. The minimum time required for regeneration, the number of calli that exhibited regeneration, and shoot growth measured in millimeters were noted. Data were subjected to analysis of variance. The means of callus weights, shoot lengths, and percentage of calli that produced shoots were separated with LSD, where appropriate, at $P$ $=0.05$.

Limiting $C W$ addition to the regeneration stage. Calli derived from 'Baker' initiated on a $\mathrm{CW}$-free medium were transferred to either $\mathrm{CW}$-free or $\mathrm{CW}$-containing regeneration medium at $15 \% \mathrm{CW}$. Calli initiated and induced to regenerate on $\mathrm{CW}$-containing medium were included as a control. Eighteen tubes were cultured per treatment. Callus induction and regeneration media were the same as described previously.

Callus induction. Coconut water did not influence the ability of explants to form callus; $>90 \%$ of the explants formed callus. Regardless of the CW content of the medium, callus initiation commenced within 7 to 10 days after culturing. Callus growth on $\mathrm{CW}$-enriched medium, however, was greater as evidenced by the higher fresh weight at the end of the 4-week callus induction phase. Because of the greater growth of callus on $\mathrm{CW}$-enriched medium, the callus induction phase could be reduced to 2 to 3 weeks to achieve sufficient callus inoculum for the regeneration phase compared to 4 weeks in the absence of CW.

A significant increase in callus fresh weight in the presence of $\mathrm{CW}$ was observed for both cultivars tested (data not shown). On the CWfree medium, callus weight of the two cultivars was similar with the addition of $\mathrm{CW}$, however, 'Baker' produced significantly more callus than 'High Pack'.

Callus growth of both cultivars depended on the concentration of CW (Fig. 1A). Although callus weight continued to increase in response to increasing levels of $\mathrm{CW}$, the
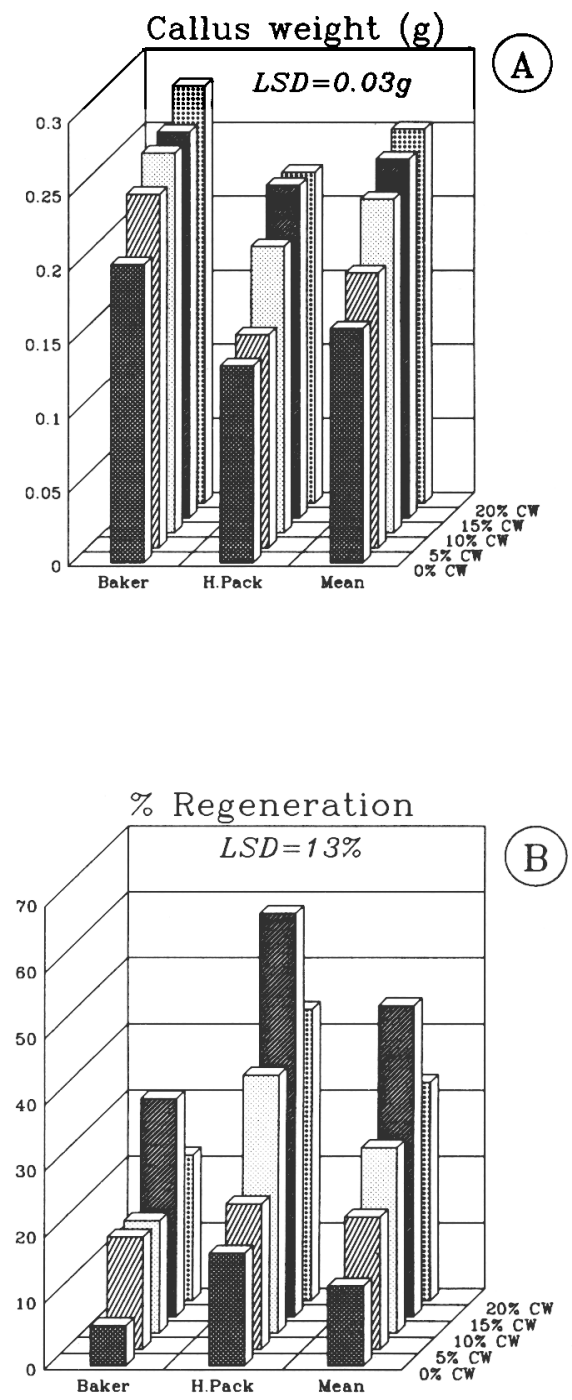

Fig 1. The response of two spinach cultivars to coconut water $(\mathrm{CW})$ at $0 \%, 5 \%, 10 \%, 15 \%$, or 20\%. (A) Callus fresh weight; (B) percentage of calli exhibiting shoot regeneration. 
rate of increase in callus weight diminished. Therefore, $10 \%$ to $15 \% \mathrm{CW}$ is recommended for spinach medium. Increasing the $\mathrm{CW}$ level to $20 \%$ gave only a slight increase, particularly with 'High Pack'.

Shoot regeneration. In the absence of $\mathrm{CW}$, calli derived from 'High Pack' regenerated at a frequency significantly higher than that exhibited by 'Baker'. This relationship also held true in the presence of CW. The percentage of calli, which regenerated shoots from 'High Pack' on CW-free medium, was not significantly different from the percentage of regeneration from 'Baker' on CWenriched medium. A significant interaction of genotype and coconut water was also observed in a study with wheat (Triticum aestivum L. em. thell) callus (Mathias and Simpson, 1986). They found that shoot development was promoted by $10 \%(\mathrm{v} / \mathrm{v}) \mathrm{CW}$ in some lines but was inhibited in others. In our study, however, shoot formation in both genotypes of spinach was promoted by $\mathrm{CW}$ addition, but the degree of increase in regeneration frequency was cultivar dependent.

The percentage of calli that regenerated shoots increased with concentration of $\mathrm{CW}$ up to $15 \%$ (Fig. 1B). With concentrations $>15 \%$, however, shoot regeneration was inhibited in both cultivars tested.

Growth of regenerated shoots of both cultivars was significantly improved as a result of CW enrichment (data not shown). Shoot length of cultivars was unaffected by CW.

Regeneration was substantially expedited with $\mathrm{CW}$ enrichment. In the absence of $\mathrm{CW}$, up to 12 weeks of culture was required before shoot formation became apparent. With $\mathrm{CW}$, however, the time required was reduced to 4 to 5 weeks. There was no definite pattern of the onset of regeneration in relation to increasing concentration of $\mathrm{CW}$.

Limiting $C W$ addition to the regeneration stage. Augmenting only the regeneration medium with $\mathrm{CW}$ was sufficient to provide a significant improvement in shoot regeneration of spinach. Calli induced on a CW-free medium exhibited a significant increase in regenerative capacity upon transfer to $\mathrm{CW}$ enriched regeneration medium. Regenerative capacity of calli induced on a CW-free medium $(33 \%)$. and callus induced on a CWenriched medium $(41 \%)$ were similar when these calli were transferred onto a CW-enriched regeneration medium. In contrast, calli grown and induced to differentiate on a CWfree medium exhibited significantly less shoot regeneration $(15 \%)$.

Although CW addition to callus induction medium is not required to achieve high regenerative capacity, when callus multiplication or rapid callus growth is desired, callus induction and continuous subculture of callus on CW-enriched medium are recommended. Regenerating calli should be maintained on CW-enriched regeneration medium if a shoot multiplication phase is used to increase the number of the regenerated shoots. A multiplication step consists of transferring the regenerating calli onto fresh regeneration medium in GA-7 Magenta ves- sels (Magenta, Chicago) that provide ample space for shoot multiplication.

Stimulation of callus growth and shoot regeneration by $\mathrm{CW}$ in two cultivars of spinach has been shown in this study. Enrichment of the culture medium with $\mathrm{CW}$ during the regeneration phase of the system would provide the same effect as enriching the media at the callus induction and shoot regeneration phases. The increase in shoot regeneration capacity of callus, the expedited callus proliferation and the shoot differentiation associated with $\mathrm{CW}$ addition would maximize potential use of this tissue culture system as a method for the rapid propagation of selected spinach plants.

\section{Literature Cited}

Al-Khayri, J. M., F.H. Huang, and T.E. Morelock. 1991a. Regeneration of spinach from leaf callus. HortScience 26:913-914.

Al-Khayri, J. M., F. H. Huang, T.E. Morelock, and T.A. Busharar. 1991b. Genotype-dependent response of spinach callus induction and shoot regeneration. Plant Sci. 78:121-127.

Mathias, R.J. and E.S. Simpson. 1986. The interaction of genotype and culture medium on the tissue culture responses of wheat (Triticum aestivum L. em. thell). Plant Cell Tissue Organ cult. 7:31-37.

Murashige, T. and F. Skoog. 1962. A revised medium for rapid growth and bioassay with tobacco tissue cultures. Physiol. Plant. 15:473497. 